\title{
CRESCIMENTO VEGETATIVO E COEFICIENTE DE CULTURA DO FEIJOEIRO RELACIONADOS A GRAUS-DIA ACUMULADOS ${ }^{1}$
}

\author{
GERSON ARAUJO DE MEDEIROS ${ }^{2}$, FLAVIO BUSSMEYER ARRUDA ${ }^{3}$, \\ EMÍLIO SAKAI ${ }^{4}$, MAMOR FUJIWARA ${ }^{5}$ e NEWTON ROBERTO BONI ${ }^{6}$
}

\begin{abstract}
RESUMO - Estudou-se o crescimento vegetativo e o coeficiente de cultura $\left(\mathrm{k}_{\mathrm{c}}\right)$ do feijoeiro (Phaseolus vulgaris $\mathrm{L}$.), relacionando-os ao índice de graus-dia acumulados $(\Sigma \mathrm{G})$. O delineamento experimental foi o inteiramente casualizado com três tratamentos e quatro repetições. Os tratamentos de densidade populacional de plantas foram: cultura semeada em evapotranspirômetros a uma densidade de 50 plantas $\mathrm{m}^{-2}$, com redução para 25 plantas $\mathrm{m}^{-2}$, quando alcançada a cobertura completa do solo; cultura semeada com densidades populacionais de 14 e 28 plantas $\mathrm{m}^{-2}$ em campo irrigado. Os parâmetros de crescimento da cultura, como a matéria seca total, a porcentagem de cobertura vegetal e o índice de área foliar mostraram uma relação altamente significativa com $\Sigma \mathrm{G}$, tendo sido afetada pelos tratamentos e pelas condições de suprimento de água. A relação entre o coeficiente de cultura basal $\left(\mathrm{k}_{\mathrm{cb}}\right)$ e $\Sigma \mathrm{G}$ foi altamente significativa para a cultura semeada nos evapotranspirômetros. No campo, o molhamento da superfície do solo após a chuva ou irrigação e o déficit hídrico moderado afetaram as correlações $\left(\mathrm{r}^{2}\right)$ entre $\mathrm{k}_{\mathrm{c}}$ e $\Sigma \mathrm{G}$. Os tratamentos não alteraram os estádios fenológicos da cultura, quando correlacionados com graus-dia acumulados.
\end{abstract}

Termos para indexação: Phaseolus vulgaris, irrigação, população de plantas, características agronômicas, cobertura vegetal, área foliar, matéria seca.

\section{VEGETATIVE GROWTH AND BEAN CROP COEFFICIENT AS RELATED TO ACCUMULATED GROWING-DEGREE-DAYS}

\begin{abstract}
The vegetative growth and the crop coefficient $\left(\mathrm{k}_{c}\right)$ for irrigated beans (Phaseolus vulgaris $\mathrm{L}$.) was studied in relation to accumulated growing-degree-days ( $\Sigma \mathrm{G})$. A completely randomized design was used with three treatments and four replicates. Population density treatments were: crop sowed in evapotranspirometers at a density of 50 plants $\mathrm{m}^{-2}$ reduced to 25 plants $\mathrm{m}^{-2}$, when the canopy achieved full ground cover; and crop sowed with population densities of 14 and 28 plants $\mathrm{m}^{-2}$ in irrigated field. Crop growth was evaluated considering dry matter, vegetative ground cover and the leaf area index. All traits had a highly significant relationship to $\Sigma \mathrm{G}$, even though plant density and water supply conditions affected them. The relationship between the basal crop coefficient $\left(\mathrm{k}_{\mathrm{cb}}\right)$ and $\Sigma \mathrm{G}$ was highly significant in the evapotranspirometers. In the field, the surface wetting after the rain or irrigation and the mild water deficits affected the correlations $\left(\mathrm{r}^{2}\right)$ between $\mathrm{k}_{\mathrm{c}}$ and $\Sigma \mathrm{G}$. The phenological events of the plants studied were not affected by the treatment, when related to accumulated growingdegree-days.
\end{abstract}

Index terms: Phaseolus vulgaris, irrigation, plant population, agronomic characters, ground cover, leaf area, dry matter.

${ }^{1}$ Aceito para publicação em 28 de julho de 1999.

${ }^{2}$ Eng. Agríc., M.Sc., Centro Regional Universitário de Espírito Santo do Pinhal, Caixa Postal 05, CEP 13990-000 Espírito Santo do Pinhal, SP.

E-mail: gerson_medeiros@creupi.br

${ }^{3}$ Eng. Agrôn., Ph.D., Centro de Ecofisiologia e Biofísica, Instituto Agronômico (IAC), Caixa Postal 28, CEP 13001-970, Campinas, São Paulo.

E-mail: farruda@cec.iac.br

${ }^{4}$ Eng. Agrôn., Dr., Centro de Ecofisiologia e Biofísica, IAC. E-mail: emilio@cec.iac.br

${ }^{5}$ Eng. Agrôn., Centro de Ecofisiologia e Biofísica, IAC.

${ }^{6}$ Eng. Agrôn., in memoriam.

\section{INTRODUÇÃO}

O crescimento da cultura do feijoeiro é fortemente afetado pelas condições mesológicas. A temperatura é um dos principais fatores ambientais no controle do desenvolvimento e produção desta planta (Arruda et al., 1980; Wallace et al., 1991).

O conceito de tempo termal, em substituição ao da contagem cronológica, tem sido utilizado desde 1730 (Wang, 1960). Segundo este conceito, as plantas desenvolvem-se à medida que se acumulam 
unidades térmicas acima de uma temperatura base, ao passo que abaixo dessa temperatura o crescimento cessa. Através do acúmulo térmico, também conhecido como graus-dia, têm-se obtido ótimas correlações com a duração do ciclo da cultura, ou com os estádios do desenvolvimento fenológico de uma dada cultivar. Por este motivo, modelos matemáticos de crescimento do feijoeiro (Gutierrez et al., 1994; Hoogenboom et al., 1994), perda de água, de solo e de nutrientes (Williams et al., 1989; Laflen et. al., 1991) e consumo de água, incorporam rotinas de simulação de crescimento da cultura, utilizando-se o conceito de graus-dia acumulados $(\Sigma \mathrm{G})$.

O consumo de água de diversas culturas também tem sido estimado a partir de relações entre o coeficiente de cultura $\left(\mathrm{k}_{\mathrm{c}}\right)$ ou coeficiente de cultura basal $\left(\mathrm{k}_{\mathrm{cb}}\right)$ a graus-dia acumulados, como verificado para a alfafa, sorgo, algodão, milho e feijão (Sammis et al., 1985; Stegman, 1988; Amos et al., 1989; Sepaskhah \& Ilampour, 1995; Nielsen \& Hinkle, 1996). Outros autores relacionaram o coeficiente de cultura do feijoeiro a parâmetros de crescimento da planta, como o índice de área foliar, os quais são simulados em modelos matemáticos de crescimento por meio da quantificação do somatório de graus-dia (Hoogenboom et al., 1994). Estas abordagens são formas de se levar em consideração o efeito da temperatura, e outros fatores ambientais, no desempenho da cultura e na sua variação de ano para ano.

O objetivo deste trabalho foi verificar a influência das condições de manejo de água e de densidade populacional de plantas sobre o crescimento vegetativo, o coeficiente de cultura, e o estádio fenológico do feijoeiro relacionados com os graus-dia acumulados.

\section{MATERIAL E MÉTODOS}

O trabalho foi desenvolvido no Núcleo Experimental de Campinas (NEC), na área de pesquisa em irrigação e drenagem do Instituto Agronômico (IAC), na longitude $47^{\circ} 04^{\prime}$ W e latitude $22^{\circ} 52^{\prime} \mathrm{S}$, num solo classificado como Latossolo Roxo distrófico A moderado, muito argiloso, unidade Barão Geraldo, de acordo com a Seção de Pedologia do IAC. As propriedades físicas e químicas selecionadas do solo (Tabela 1) foram analisadas de acordo com a metodologia descrita por Camargo et al. (1986).
TABELA 1. Análise física e de fertilidade do Latossolo Roxo, unidade Barão Geraldo, Campinas, SP.

\begin{tabular}{lccc}
\hline Propriedades & \multicolumn{3}{c}{ Profundidade $(\mathrm{cm})$} \\
\cline { 2 - 4 } & $0-20$ & $20-40$ & $40-60$ \\
\hline Areia grossa (\%) & 16,7 & 13,2 & \\
Areia fina (\%) & 17,1 & 14,3 & \\
Silte (\%) & 5,0 & 5,0 & \\
Argila (\%) & 61,2 & 67,5 & \\
Densidade do solo $\left(\mathrm{g} \mathrm{cm}^{-3}\right)$ & 1,22 & 1,13 & \\
Densidade de partículas $\left(\mathrm{g} \mathrm{cm}^{-3}\right)$ & 2,73 & 2,76 & \\
$\mathrm{P}\left(\mathrm{mg} \mathrm{dm}^{-3}\right)$ & 41,0 & 11,0 & 4,0 \\
$\mathrm{M} . \mathrm{O} .(\%)$ & 3,9 & 2,7 & 1,9 \\
$\mathrm{pH}\left(\mathrm{CaCl}_{2}\right)$ & 6,0 & 5,4 & 5,3 \\
$\mathrm{~K}^{+}\left(\mathrm{mmol}_{\mathrm{c}} \mathrm{dm}^{-3}\right)$ & 4,4 & 1,1 & 0,3 \\
$\mathrm{Ca}{ }^{2+}\left(\mathrm{mmol}_{\mathrm{c}} \mathrm{dm}^{-3}\right)$ & 67 & 37 & 28 \\
$\mathrm{Mg}^{2+}\left(\mathrm{mmol}_{\mathrm{c}} \mathrm{dm}^{-3}\right)$ & 28 & 19 & 17 \\
$\mathrm{H}^{+}+\mathrm{Al}^{3+}\left(\mathrm{mmol}_{\mathrm{c}} \mathrm{dm}^{-3}\right)$ & 23 & 28 & 25 \\
$\mathrm{~S}\left(\mathrm{mmol}_{\mathrm{c}} \mathrm{dm}^{-3}\right)$ & 99 & 57 & 45 \\
$\mathrm{~T}\left(\mathrm{mmol}_{\mathrm{c}} \mathrm{dm}^{-3}\right)$ & 122 & 85 & 70 \\
$\mathrm{~V}(\%)$ & 81 & 67 & 64 \\
\hline
\end{tabular}

A Fig. 1 apresenta os parâmetros climáticos ocorridos durante o ciclo da cultura no período de 26 de agosto a 15 de novembro de 1994. A Tabela 2 apresenta os valores mensais das normais climatológicas e dos observados durante o período do ensaio em Campinas, SP, segundo dados fornecidos pelo Centro de Ecofisiologia e Biofísica do IAC.

Os valores da Tabela 2 indicam que o período do ensaio foi mais seco e quente do que nas condições apresentadas pelas normais climatológicas. Nas fases de crescimento ativo e florescimento, durante os meses de setembro e outubro, as chuvas foram de apenas $51,9 \mathrm{~mm}$; e do total precipitado, de $181 \mathrm{~mm}$ ao longo do ciclo da cultura, $49 \%$ $(88,5 \mathrm{~mm})$ ocorreu na última semana do experimento. A temperatura média do ar, de 26 de agosto a 15 de novembro de 1994 , foi de $24,2^{\circ} \mathrm{C}$, sendo a média das temperaturas mínimas de $16,3^{\circ} \mathrm{C}$, e a média das temperaturas máximas, de $31,0^{\circ} \mathrm{C}$.

A demanda evaporativa média durante o ensaio foi de 5,9 $\mathrm{mm} \mathrm{dia}^{-1}$, determinada pelo método de Penman. A maior taxa média evaporativa ocorreu no período de 1 a 20/10/1994 (36 a 56 dias após emergência), atingindo $6,8 \mathrm{~mm} \mathrm{dia}^{-1}$, durante a fase de pleno florescimento.

A má distribuição de chuvas e as altas temperaturas não permitiriam uma produção econômica da cultura do feijoeiro sem o emprego da irrigação.

A cultivar Carioca 80-SH foi semeada com diferentes densidades de semeadura, em um delineamento inteiramente casualizado, com três tratamentos e quatro repetições, sob duas condições distintas de manejo de água: a) no campo, com aplicação de água por sistema de irrigação por aspersão convencional, utilizando-se dois espaçamentos 
$(0,5$ e $1,0 \mathrm{~m})$, com populações de 28 e 14 plantas $\mathrm{m}^{-2}$ respectivamente, totalizando oito parcelas de $13 \times 13 \mathrm{~m}$; b) em evapotranspirômetros de nível freático constante, com um espaçamento de $27,5 \mathrm{~cm}$, correspondendo a uma
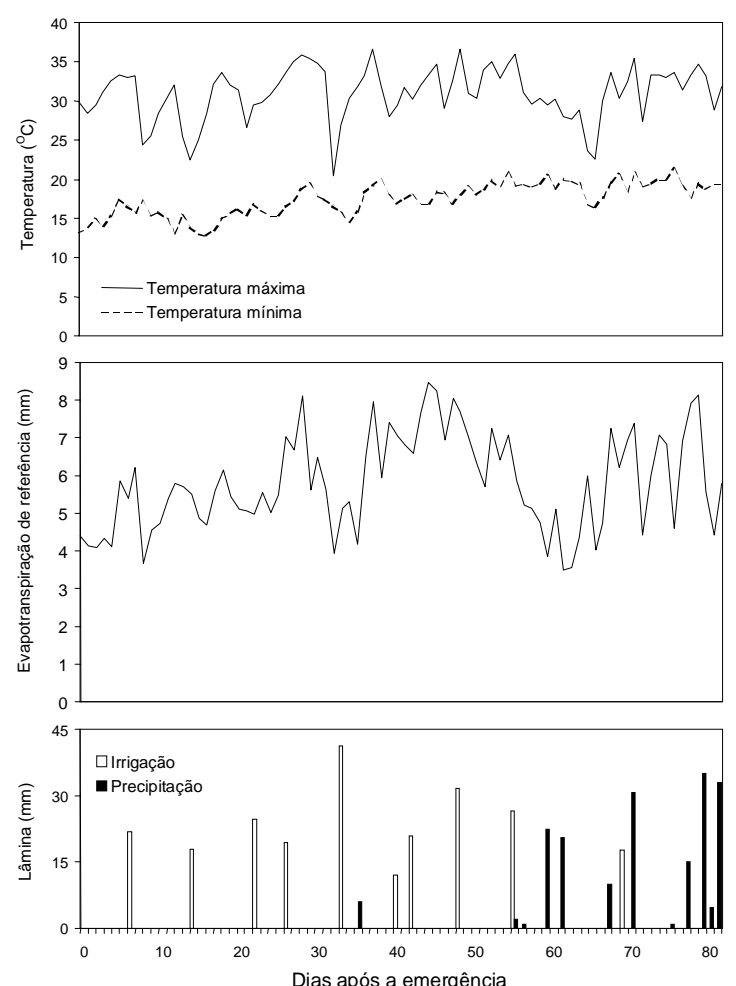

FIG. 1. Condições climáticas no período do ensaio com feijoeiro irrigado, observadas em Campinas, 1994. Dados do posto meteorológico instalado no Núcleo Experimental de Campinas, sob condições padronizadas, e gerenciado pelo Centro de Ecofisiologia Vegetal. população de 50 plantas $\mathrm{m}^{-2}$. Aos 25 dias após a emergência (DAE), quando se atingiu a cobertura total do solo, fez-se um desbaste, reduzindo-se a população para 25 plantas $\mathrm{m}^{-2}$.

Os evapotranspirômetros eram compostos por reservatórios com 1.000 L de capacidade e uma área de exposição de $1,41 \mathrm{~m}^{2}$, equipados com um sistema de alimentação de água para manter um nível freático a $0,45 \mathrm{~m}$ abaixo da superfície do solo. Eles foram cobertos temporariamente durante os eventos de chuva, evitando-se o molhamento da superfície do solo. O funcionamento e as adaptações realizadas nos evapotranspirômetros para atender às condições necessárias para a obtenção de $\mathrm{k}_{\mathrm{cb}}$ são descritas em Medeiros \& Arruda (1998).

A semeadura no campo e nos evapotranspirômetros foi realizada em 16/8/1994, e a emergência ocorreu em 26/8/1994. A colheita, em ambos os casos, foi realizada em 15/11/1994.

Para a determinação dos parâmetros de crescimento (de plantas), foram tomadas amostras de $4 \mathrm{~m}$ de linha de planta por tratamento no campo irrigado, sendo $1 \mathrm{~m}$ por parcela. A matéria seca total (MS) e a sua partição foram obtidas após secagem em estufa a $60^{\circ} \mathrm{C}$, por um período mínimo de 48 horas. O índice de área foliar (IAF) foi determinado pela relação entre a área foliar medida no aparelho Lambda-Licor, modelo LI 3000, e a área ocupada pela cultura. Determinou-se a porcentagem de cobertura vegetal (\%CVeg) pelo método da fotografia (Arruda, 1984), considerando-se oito fotografias por tratamento, equivalente, aproximadamente, ao mesmo tamanho da área amostral de plantas.

O coeficiente de cultura foi obtido por meio da relação: $\mathrm{k}_{\mathrm{c}}=\frac{\mathrm{ET}}{\mathrm{ET}_{\mathrm{o}}}$,

onde ET é a evapotranspiração real (mm) no período considerado, e $\mathrm{ET}_{\mathrm{o}}$, a evapotranspiração de referência $(\mathrm{mm})$, calculada pelo método de Penman, adaptado para o Estado de São Paulo por Villa Nova \& Ometto (1981).

O coeficiente de cultura basal $\left(\mathrm{k}_{\mathrm{cb}}\right)$, conforme definido por Wright (1981), corresponde ao $\mathrm{k}_{\mathrm{c}}$ obtido quando a

TABELA 2. Normais climatológicas e valores médios mensais observados no ano de 1994 em Campinas, SP, segundo dados fornecidos pelo Centro de Ecofisiologia e Biofísica do Instituto Agronômico.

\begin{tabular}{|c|c|c|c|c|c|c|c|c|c|c|}
\hline \multirow[t]{2}{*}{ Mês } & \multicolumn{2}{|c|}{ Precipitação (mm) } & \multicolumn{2}{|c|}{$\begin{array}{l}\text { Umidade } \\
\text { relativa (\%) }\end{array}$} & \multicolumn{2}{|c|}{$\begin{array}{l}\text { Temperatura } \\
\text { mínima }\left({ }^{\circ} \mathrm{C}\right)\end{array}$} & \multicolumn{2}{|c|}{$\begin{array}{l}\text { Temperatura } \\
\text { média }\left({ }^{\circ} \mathrm{C}\right)\end{array}$} & \multicolumn{2}{|c|}{$\begin{array}{l}\text { Temperatura } \\
\text { máxima }\left({ }^{\circ} \mathrm{C}\right)\end{array}$} \\
\hline & Normal & 1994 & Normal & 1994 & Normal & 1994 & Normal & 1994 & Normal & 1994 \\
\hline Ago. & 37,4 & 0,0 & 64,6 & 62,3 & 13,2 & 12,9 & 18,9 & 19,3 & 26,4 & 27,0 \\
\hline Set. & 65,6 & 6,0 & 65,5 & 53,7 & 14,9 & 15,7 & 20,3 & 23,2 & 27,2 & 30,1 \\
\hline Out. & 123,6 & 45,9 & 69,2 & 60,7 & 16,3 & 18,5 & 21,3 & 24,7 & 28,0 & 31,1 \\
\hline Nov. & 137,5 & 177,8 & 70,8 & 64,4 & 17,3 & 18,3 & 22,1 & 24,0 & 28,6 & 29,7 \\
\hline
\end{tabular}


evaporação do solo é mínima, mas a umidade na zona das raízes adequada, não limitando nem o crescimento da planta nem a transpiração. O seu valor foi calculado pela equação 1, observando-se o consumo médio de água ocorrido no evapotranspirômetro num período de cinco dias, tempo comumente utilizado em estudos desta natureza com relação ao feijoeiro no Brasil (Encarnação, 1980; Pavani, 1985).

A evapotranspiração real (ET) do feijoeiro foi estimada por meio do balanço hídrico de campo, descrito por Reichardt (1985), para um volume de controle com 0,40 m de profundidade, correspondente a uma concentração superior a 90\% de raízes (Pires et al., 1991). Neste balanço, desprezaram-se os períodos durante os quais algumas chuvas prolongadas ocorreram, como aquelas do final do ciclo, em virtude do aumento da importância relativa do termo drenagem profunda. Nos evapotranspirômetros, estes mesmos períodos foram desprezados, pois a cobertura de plástico interferiu no consumo de água pelas plantas do feijoeiro.

Os valores de graus-dia foram calculados por meio da seguinte expressão (Ometto, 1981):

$\mathrm{G}=\frac{\left(\mathrm{T}_{\text {máx }}+\mathrm{T}_{\text {min }}\right)}{2}-\mathrm{T}_{\text {base }}$,

onde, $\mathrm{T}_{\text {máx }}$ é a temperatura máxima do ar $\left({ }^{\circ} \mathrm{C}\right), \mathrm{T}_{\text {mín }}$ é a temperatura mínima do ar $\left({ }^{\circ} \mathrm{C}\right), \mathrm{T}_{\text {base }}$ é a temperatura abaixo da qual as plantas não se desenvolvem, tendo sido adotado o valor de $10^{\circ} \mathrm{C}$ (Kish \& Ogle, 1980).

\section{RESULTADOS E DISCUSSÃO}

\section{Crescimento e produção final}

A Fig. 2 apresenta as relações entre a \%CVeg e o IAF com o somatório de graus-dia para o crescimento do feijoeiro no campo, em duas populações de plantas.

Aos resultados obtidos ajustaram-se equações polinomiais com alto grau de significância. No tratamento de 14 plantas $\mathrm{m}^{-2}$, as equações obtidas foram:

$\%$ CVeg $=1,332+0,026 \sum G+1,68 * 10^{-4} \sum G^{2}-1,27 * 10^{-7} \sum G^{3}$, $\mathrm{n}=18, \mathrm{r}^{2}=0,99 * * \quad \mathrm{e}$

IAF $=0,100-1,95 * 10^{-3} \sum \mathrm{G}+1,54 * 10^{-5} \sum \mathrm{G}^{2}-1,10 * 10^{-8} \sum \mathrm{G}^{3}$, $\mathrm{n}=16, \mathrm{r}^{2}=0,98 * *$

No tratamento de 28 plantas $\mathrm{m}^{-2}$, obtiveram-se as seguintes relações:

$\% \mathrm{CVeg}=5,225+0,012 \sum \mathrm{G}+4,30 * 10^{-4} \sum \mathrm{G}^{2}-3,66 * 10^{-7} \sum \mathrm{G}^{3}$, $\mathrm{n}=18, \mathrm{r}^{2}=0,97 * * \mathrm{e}$
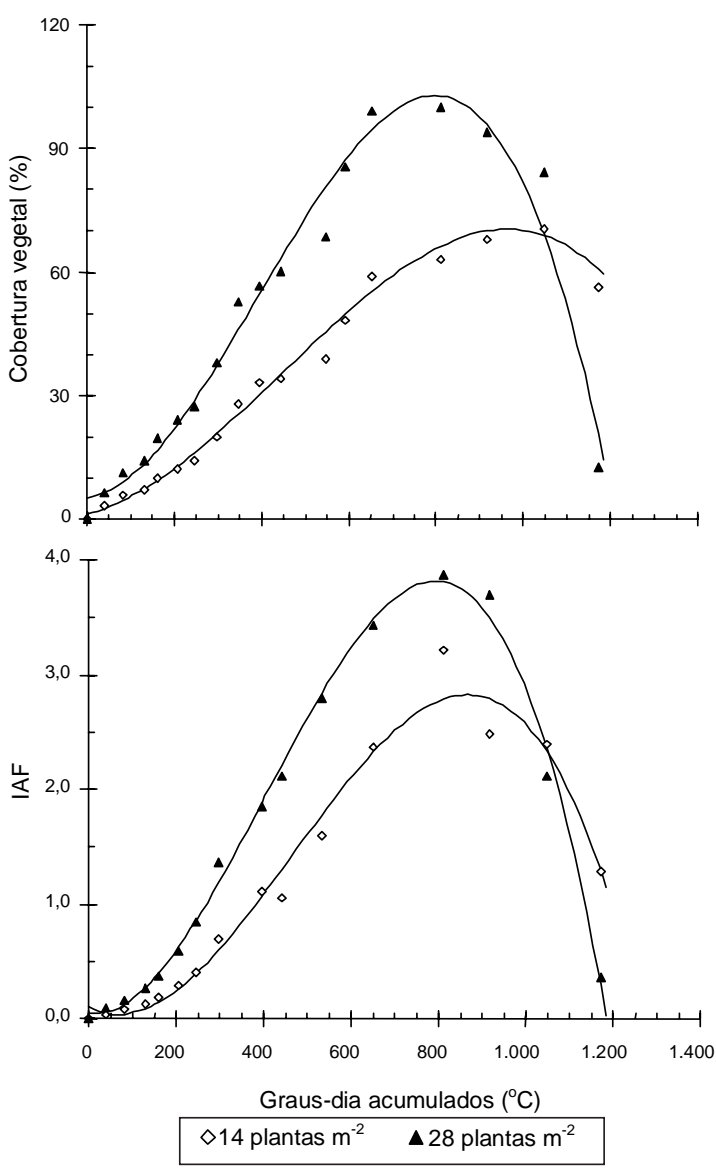

FIG. 2. Porcentagem da cobertura vegetal e índice de área foliar (IAF) em razão do somatório de graus-dia referentes ao crescimento da cultura do feijoeiro, com densidades de semeadura de 14 e 28 plantas $\mathrm{m}^{-2}$, em condições de campo, em Campinas, 1994.

IAF $=0,046-4,97 * 10^{-4} \sum \mathrm{G}+1,92 * 10^{-5} \sum \mathrm{G}^{2}-1,59 * 10^{-8} \sum \mathrm{G}^{3}$, $\mathrm{n}=16, \mathrm{r}^{2}=0,99 * *$.

Os resultados da cobertura vegetal, em razão do somatório de graus-dia obtidos nos evapotranspirômetros, são apresentados na Fig. 3. Na Fig. 3A, é mostrada a variação da \%CVeg, na fase vegetativa, até o momento do desbaste, quando o acúmulo foi de 300 graus-dia. A relação altamente significativa encontrada foi:

$\%$ CVeg $=7,264+0,0327 \sum \mathrm{G}+9,55 * 10^{-4} \sum \mathrm{G}^{2}$, $\mathrm{n}=7, \quad \mathrm{r}^{2}=0,98 * *$.

Na Fig. 3B, elaborada para uma densidade de semeadura de 25 plantas $\mathrm{m}^{-2}$, considerou-se que a 


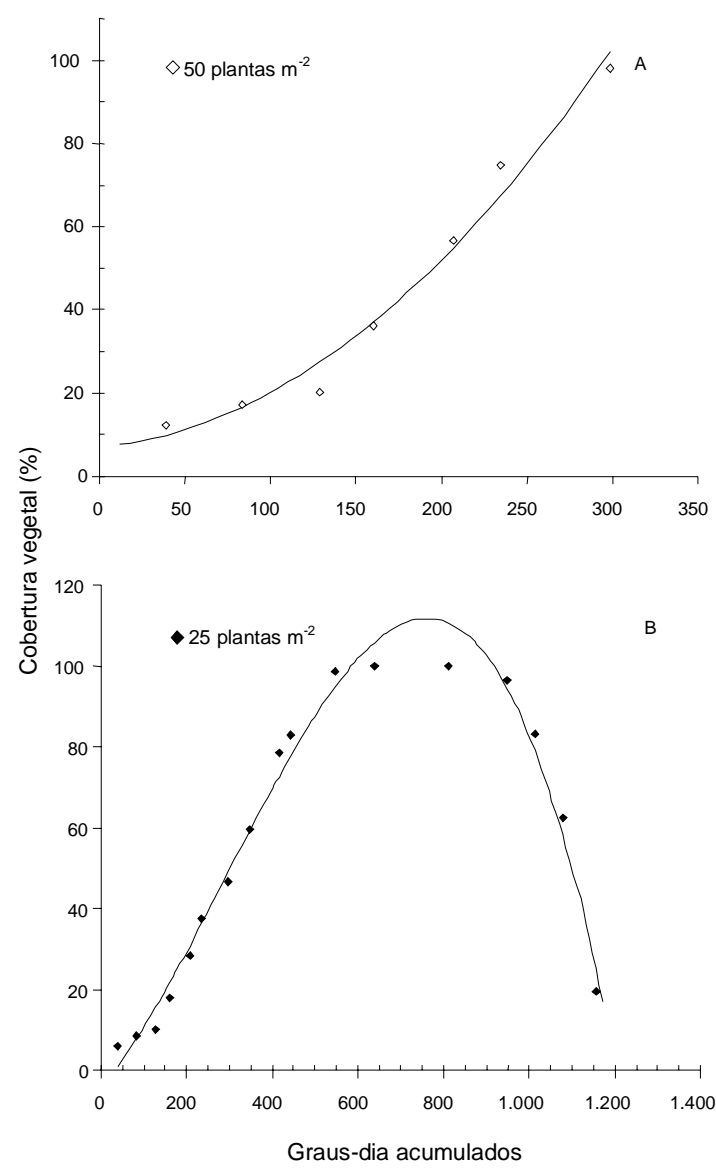

FIG. 3. Porcentagem da cobertura vegetal em razão do somatório de graus-dia para o crescimento da cultura do feijoeiro, com densidades de semeadura de 25 e 50 plantas $\mathrm{m}^{-2}$, semeada em evapotranspirômetros, em Campinas, 1994.

\% CVeg correspondeu à metade da densidade determinada na cultura de 50 plantas $\mathrm{m}^{-2}$ até o momento do desbaste (aos 25 DAE). Tal procedimento foi adotado porque houve um rápido crescimento vegetativo no tratamento de 50 plantas $\mathrm{m}^{-2}$, com a cobertura vegetal da cultura variando de $50 \%$ a $100 \%$ na semana que antecedeu o desbaste. Conseqüentemente, assumiu-se que o efeito da competição por luz ocorreu num curto intervalo de tempo, e como as condições de suprimento de água foram adequadas, admitiu-se que o crescimento da planta não tenha sido significativamente afetado devido ao adensamento até o momento do desbaste.

No tratamento de 25 plantas $\mathrm{m}^{-2}$, nos evapotranspirômetros, obteve-se a seguinte relação:

$\%$ CVeg $=-4,331+0,127 \sum \mathrm{G}+2,67 * 10^{-4} \sum \mathrm{G}^{2}-3,07 * 10^{-7} \sum \mathrm{G}^{3}$, $\mathrm{n}=18, \mathrm{r}^{2}=0,98 * *$

O fechamento completo da cobertura vegetal foi observado nos tratamentos de 25 e 50 plantas $\mathrm{m}^{-2}$ (evapotranspirômetros) quando acumulados 638 e 298 graus-dia, respectivamente (Fig. 3). No tratamento de 28 plantas $\mathrm{m}^{-2}$, o fechamento ocorreu somente quando foram acumulados 812 graus-dia (Fig. 2).

O acúmulo da matéria seca total (MS) e das partes da planta, em razão do somatório de graus-dia nos tratamentos de 14 e 28 plantas $\mathrm{m}^{-2}$, é apresentado na Fig. 4. Nesta figura, observa-se que o acúmulo da matéria seca das folhas e caule praticamente estabilizou-se por volta de 810 graus-dia. As curvas de acúmulo de matéria seca total e de partes da planta apresentam muita similaridade entre os tratamentos, o que indica que as populações pouco afetaram o padrão de partição de matéria seca da cultura. Quanto à produção, contudo, o momento de estabilização do acúmulo da matéria seca total diferiu entre os tratamentos. No tratamento de maior densidade, o início da estabilização da matéria seca total ocorreu aos $950 \Sigma \mathrm{G}$, enquanto no de 14 plantas $\mathrm{m}^{-2}$ ocorreu aos $1.050 \Sigma \mathrm{G}$ (Fig. 4). A diferença é devida à antecipação do período de senescência das folhas inferiores, verificado no tratamento de maior densidade populacional, causada pela menor penetração de luz em relação ao tratamento de 14 plantas $\mathrm{m}^{-2}$. Villacorta et al. (1990), em ensaio de feijão irrigado no Paraná, com a cultivar Carioca, semeada a uma baixa densidade de 10 plantas $\mathrm{m}^{-2}$, observaram que o declínio da matéria seca total também ocorreu tardiamente aos $1.100 \Sigma \mathrm{G}$.

O período de maior demanda por assimilados, pelos órgãos reprodutivos, ocorreu entre 655 e 1.050 graus-dia (Fig. 4), correspondendo ao período de 49 a 75 DAE.

As relações desenvolvidas para descrever o acúmulo de matéria seca do feijoeiro em função dos graus-dia acumulados, desde a emergência até o final do acúmulo de matéria seca total, forneceram 


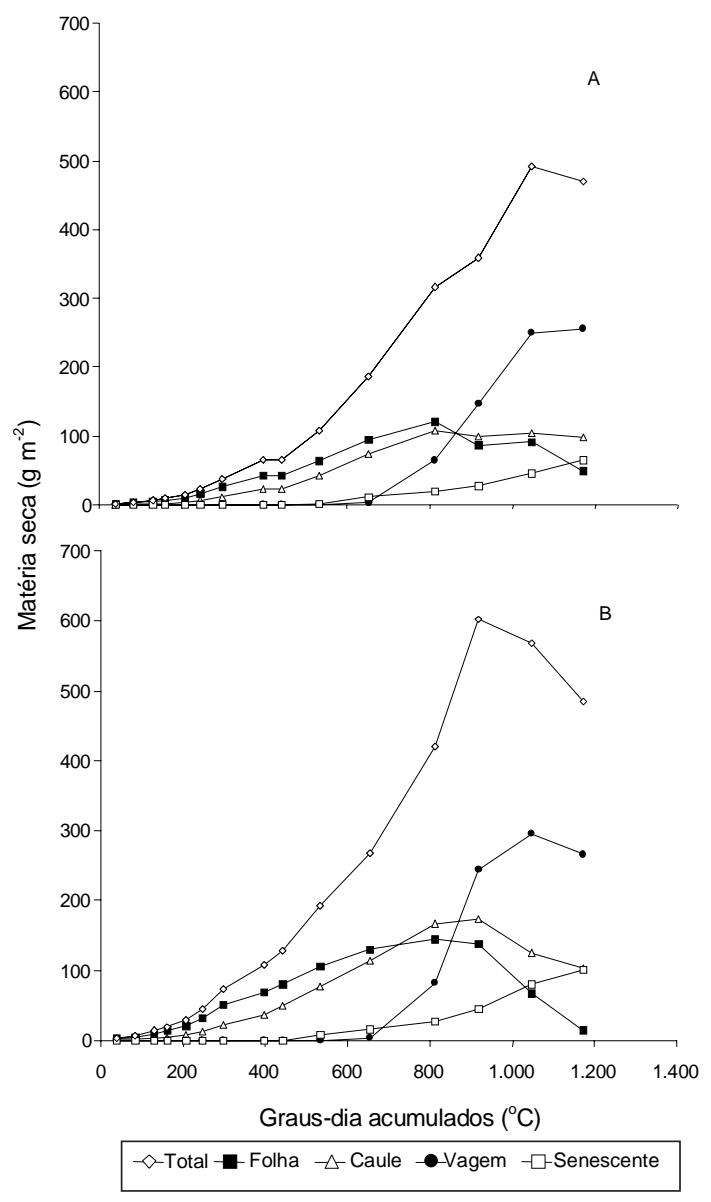

FIG. 4. Matéria seca total e sua distribuição por partes, em razão do somatório de graus-dia para o crescimento da cultura do feijoeiro, com densidades de semeadura de 14 (A) e 28 (B) plantas $\mathbf{m}^{-2}$, em condições de campo, em Campinas, 1994.

correlações altamente positivas $(\mathrm{P}<0,01)$ nos tratamentos de campo.

O tratamento de 14 plantas $\mathrm{m}^{-2}$ forneceu a seguinte relação entre MS e $\Sigma \mathrm{G}$ até 1.050 graus-dia:

$\mathrm{MS}=3,177-0,044 \sum \mathrm{G}+0,00049 \sum \mathrm{G}^{2}$,

$\mathrm{n}=14, \mathrm{r}^{2}=0,99 * *$

No tratamento de 28 plantas $\mathrm{m}^{-2}$, obteve-se uma relação similar à anterior, até 950 graus-dia:

MS $=10,906-0,063 \sum G+0,00074 \sum G^{2}$, $\mathrm{n}=13, \mathrm{r}^{2}=0,99 * *$

A média da taxa máxima de acúmulo de matéria seca total da cultura ajustada na fase linear, de 530 a $1.050 \Sigma \mathrm{G}$, nos tratamentos de densidade de semeadurade $14 \mathrm{e} 28$ plantas $\mathrm{m}^{-2}$, foi de $0,73 \mathrm{e} 0,84 \mathrm{~g} \mathrm{~m}^{-2}$ graus-dia ${ }^{-1}$, respectivamente. Estes valores observados correspondem à taxa de crescimento da cultura (TCC) de 10,9 e $12,7 \mathrm{~g} \mathrm{~m}^{-2} \mathrm{dia}^{-1}$, respectivamente. A diferença é explicada pela maior cobertura vegetal do solo, ao longo do período, no tratamento de maior população, e portanto, com maior interceptação da radiação e taxa fotossintética (Arruda, 1987).

Nos evapotranspirômetros, aos 25 DAE, quando foram acumulados 298 graus-dia, foram obtidos $157 \mathrm{~g} \mathrm{~m}^{-2}$ de matéria seca total para a população de 50 plantas $\mathrm{m}^{-2}$. A estimativa de $78,5 \mathrm{~g} \mathrm{~m}^{-2} \mathrm{em}$ relaço à população de 25 plantas $\mathrm{m}^{-2}$, nesta data superou os $38 \mathrm{~g} \mathrm{~m}^{-2}$ e $73 \mathrm{~g} \mathrm{~m}^{-2}$ dos tratamentos de campo, com 14 e 28 plantas $\mathrm{m}^{-2}$, respectivamente. Na colheita, aos 82 DAE, ou $1.170 \Sigma \mathrm{G}$, o valor médio de matéria seca da parte aérea atingiu $725 \mathrm{~g} \mathrm{~m}^{-2}$ na população de 25 plantas $\mathrm{m}^{-2}$ nos evapotranspirômetros, enquanto no tratamento de 28 plantas $\mathrm{m}^{-2}$ no campo, o máximo valor atingido foi de $602 \mathrm{~g} \mathrm{~m}^{-2}$, aos 67 DAE.

A taxa de crescimento médio da cultura nos evapotranspirômetros, no período de 25 a 82 DAE, ou de 300 a 1.170 graus-dia acumulados, apresentou um valor de 11,4 $\mathrm{g} \mathrm{m}^{-2} \mathrm{dia}^{-1}$, correspondendo a $0,62 \mathrm{~g} \mathrm{~m}^{-2}$ graus-dia- ${ }^{-1}$. Esta taxa de crescimento da cultura em base de temperatura foi menor do que a apresentada no tratamento de 28 plantas $\mathrm{m}^{-2}$; porém, a duração da área foliar verde, conseqüência do atraso na senescência, resultou em maior produção final da cultura nos evapotranspirômetros.

Os resultados da avaliação final da produção e de seus componentes encontram-se na Tabela 3 . Não houve diferença significativa na produção por área entre os tratamentos de densidade populacional de 14 e 28 plantas $\mathrm{m}^{-2}$ no campo, na comparação das médias feita pelo teste de Duncan, $5 \%$ de probabilidade. Contudo, houve diferença significativa na produção de grãos por planta entre estes tratamentos de densidade populacional.

A menor produção por planta do tratamento de 28 plantas $\mathrm{m}^{-2}$ foi compensado por sua maior densidade populacional em relação ao tratamento de 14 plantas $\mathrm{m}^{-2}$. 
TABELA 3. Peso seco final da produção de grãos de feijão e de seus componentes, em diferentes densidades populacionais, no campo e nos evapotranspirômetros, em Campinas, 1994 1.

\begin{tabular}{|c|c|c|c|c|c|c|c|}
\hline \multirow{2}{*}{$\begin{array}{l}\text { Tratamento } \\
\left(\text { plantas } \mathrm{m}^{-2}\right)\end{array}$} & \multirow{2}{*}{$\begin{array}{c}\text { Vagens } \\
\text { por planta } \\
\left(\mathrm{n}^{\mathrm{o}}\right)\end{array}$} & \multirow{2}{*}{$\begin{array}{c}\text { Grãos por } \\
\text { vagem } \\
\left(\mathrm{n}^{\mathrm{o}}\right)\end{array}$} & \multirow{2}{*}{$\begin{array}{c}\text { Peso } 500 \\
\text { sementes } \\
(\mathrm{g})\end{array}$} & \multicolumn{2}{|c|}{ Produção de grãos } & \multirow{2}{*}{$\begin{array}{c}\text { Matéria } \\
\text { seca total } \\
\left(\mathrm{g} \mathrm{m}^{-2}\right)\end{array}$} & \multirow{2}{*}{$\begin{array}{c}\text { Indice de } \\
\text { colheita } \\
(\%)\end{array}$} \\
\hline & & & & $\left(\right.$ g planta $\left.^{-1}\right)$ & $\left(\mathrm{g} \mathrm{m}^{-2}\right)$ & & \\
\hline 14 (campo) & $17 \mathrm{a}$ & $4,4 a$ & $100,6 a$ & $14,6 a$ & $192,5 b$ & $469,3 b$ & $41,0 \mathrm{a}$ \\
\hline 28 (campo) & $10 \mathrm{~b}$ & $3,8 \mathrm{a}$ & $102,0 \mathrm{a}$ & $7,2 \mathrm{~b}$ & $203,0 b$ & $484,1 b$ & $41,9 \mathrm{a}$ \\
\hline 25 (evap.) & $16 \mathrm{a}$ & $4,1 \mathrm{a}$ & $100,5 a$ & $13,0 \mathrm{a}$ & $288,0 \mathrm{a}$ & $752,3 a$ & $37,8 \mathrm{a}$ \\
\hline
\end{tabular}

${ }^{1}$ Médias seguidas de mesma letra, nas colunas, não diferem entre si, a 5\% de probalidade pelo teste Ducan.

Os resultados de número de vagens por planta justificam a maior produção do tratamento de menor densidade de plantio, apesar de o número de grãos por vagem não ter diferido significativamente entre estes tratamentos (Tabela 3).

O período de florescimento e formação de vagens coincidiu com a época de ocorrência das maiores taxas de evapotranspiração de referência do ensaio. É possível que as plantas de maior população (28 plantas $\mathrm{m}^{-2}$ ) tenham sofrido estresse hídrico moderado, afetando o número final de vagens por planta.

Os resultados de produção por área e por planta do tratamento de 25 plantas $\mathrm{m}^{-2}$, em comparação com os demais, não seguiram o comportamento geral sugerido por Gutierrez et al. (1994) de aumento do número de vagens por planta e redução da produção por área, à medida que aumenta a densidade populacional.

O desempenho, por planta, deste tratamento, foi ligeiramente inferior, porém não significativamente, em relação ao tratamento de 14 plantas $\mathrm{m}^{-2}$, apesar da maior competição por luz. Este fato levou a uma diferença significativa nos resultados de produção por área da cultura conduzida nos evapotranspirômetros, em relação aos tratamentos de campo irrigado, superando em 49,6 e $41,9 \%$ os tratamentos de 14 e 28 plantas $\mathrm{m}^{-2}$, respectivamente.

Quanto ao índice de colheita (IC) da Tabela 3, houve boa conversão da matéria seca total em produção, próximo a 40\%. Nos evapotranspirômetros, o valor inferior do IC indica que as melhores condições de umidade nos evapotranspirômetros favoreceram o maior desenvolvimento vegetativo da planta, principalmente da parte aérea, com vegetação visivelmente mais exuberante e massa verde total.
O desempenho da cultura semeada nos evapotranspirômetros, em termos de produção, foi superior ao das culturas semeadas em condições de lavoura irrigada. Entre os principais fatores responsáveis por esta diferença de produção, pode-se citar a aplicação uniforme de água, mantendo-se potenciais de água no solo superiores a $-10 \mathrm{kPa}$ a $0,05 \mathrm{~m}$ de profundidade, durante todo o ciclo, e inexistência de problemas de compactação (Medeiros \& Arruda, 1998). Wang (1960) ressalta que a abordagem de índices térmicos não leva em conta uma série de fatores que interferem nos processos de crescimento da planta, tais como a umidade do solo, déficit de pressão de vapor, entre outros, e, como evidenciado neste trabalho, a população de plantas e conseqüente cobertura vegetal.

Contudo, apesar das diferenças de produção de matéria seca observadas, a emergência, o florescimento, o período de enchimento de grãos e a duração do ciclo foram similares em relação a todos os tratamentos (Tabela 4). Pode-se concluir que a duração das fases de desenvolvimento não foram afetadas pelos tratamentos, o que demonstra a confiabilidade da abordagem de graus-dia para a determinação dos estádios fenológicos da cultura.

\section{Coeficiente de cultura}

Os resultados de coeficiente de cultura, a partir do balanço hídrico, tanto nas condições de campo irrigado, como em evapotranspirômetros, apresentaram relações altamente significativas com o somatório de graus-dia $(\mathrm{P}<0,01)$.

As relações entre $\mathrm{k}_{\mathrm{c}}$ e graus-dia acumulados para os tratamentos foram as seguintes: 
TABELA 4. Tempo termal das fases fenológicas do feijoeiro, cultivar Carioca 80-SH, semeado nas populações de 14, 25 e 28 plantas $\mathrm{m}^{-2}$, em Campinas, 1994.

\begin{tabular}{lc}
\hline Fase fenológica & Graus-dia \\
\hline 1 $^{\text {a }}$ folha trifoliada & 94 \\
$3^{\mathrm{a}}$ folha trifoliada & 246 \\
Início do florescimento & 532 \\
Início da formação da vagem & 653 \\
Enchimento do grão & 812 \\
Colheita & 1.155 \\
\hline
\end{tabular}

Tratamento de 14 plantas $\mathrm{m}^{-2}$ :

$\mathrm{k}_{\mathrm{c}}=0,180+1,57 * 10^{-3} \sum \mathrm{G}-1,04 * 10^{-6} \sum \mathrm{G}^{2}$,

$\mathrm{n}=14, \mathrm{r}^{2}=0,81 * *$

Tratamento de 28 plantas $\mathrm{m}^{-2}$ :

$\mathrm{k}_{\mathrm{c}}=0,0662+3,07 * 10^{-3} \sum \mathrm{G}-2,38 * 10^{-6} \sum \mathrm{G}^{2}$,

$\mathrm{n}=16, \mathrm{r}^{2}=0,78 * *$

Tratamento de 50 plantas $\mathrm{m}^{-2}$ :

$\mathrm{k}_{\mathrm{cb}}=0,355-0,00126 \sum \mathrm{G}+1,33 * 10^{-5} \sum \mathrm{G}^{2}$,

$\mathrm{n}=17, \mathrm{r}^{2}=0,99 * *$

Apesar das correlações significativas obtidas, a análise de fatores como a influência da cobertura vegetal se faz necessária para explicar a variabilidade do coeficiente de cultura, ao longo do ciclo. As Figs. 5 e 6 apresentam a variação de $k_{c}$ e da \%CVeg com relação ao somatório de graus-dia, ao longo do ciclo da cultura do feijoeiro irrigado.

A cobertura vegetal foi escolhida como parâmetro de desenvolvimento da planta pelo fato de ter sido medida em todos os tratamentos, e por ser recomendada por alguns autores a fim de parametrizar o coeficiente de cultura (Adams et al., 1976; Medeiros, 1996).

Observa-se, na Fig. 5, que o comportamento da curva ajustada de $\mathrm{k}_{\mathrm{c}}$ em razão do somatório de graus-dia, é semelhante ao verificado na curva de cobertura vegetal, principalmente no tratamento de 28 plantas $\mathrm{m}^{-2}$ e na cultura semeada nos evapotranspirômetros (Fig. 6). Este fato demonstra que o coeficiente de cultura pode ser melhor relacionado a parâmetros de crescimento da cultura, como a \%CVeg, do que a índices térmicos como graus-dia de crescimento acumulados, como mostrado em Medeiros (1996).

Outro importante aspecto a ser verificado na Fig. 5 refere-se ao efeito do manejo de água sobre os resul-

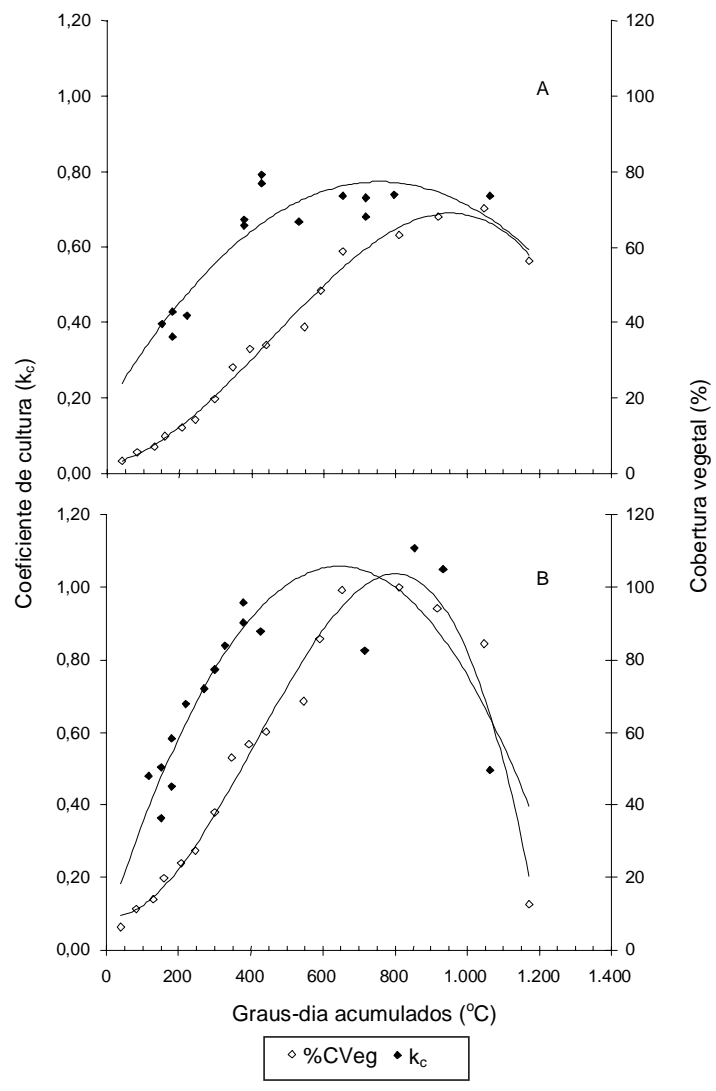

FIG. 5. Coeficiente de cultura $\left(k_{c}\right)$ e porcentagem de cobertura vegetal (\% CVeg) da cultura do feijoeiro em razão dos graus-dia acumulados, nos tratamentos de 14 (A) e 28 (B) plantas $\mathbf{m}^{-2}$, em Campinas, 1994.

tados de coeficiente de cultura. Os valores observados de $\mathrm{k}_{\mathrm{c}}$ no campo são médias ocorridas no período entre amostras e, de acordo com o critério de Wright (1981), sofreram a influência de chuva ou irrigação. Quando há o molhamento da superfície do solo, os valores de $\mathrm{k}_{\mathrm{c}}$ sofrem um incremento, o que faz com que atinja seus maiores índices em curtos períodos imediatamente posteriores à irrigação ou precipitação (Medeiros, 1996). Neste caso, os valores médios de $\mathrm{k}_{\mathrm{c}}$ são superiores ou iguais a $\mathrm{k}_{\mathrm{cb}}$. Observou-se, também, no ambiente de campo, a ocorrência de estresse hídrico moderado, com redução da transpiração, principalmente após o recobrimento total do solo pela cultura quando a demanda hídrica foi maior. Conseqüentemente, apesar de a tensão de 


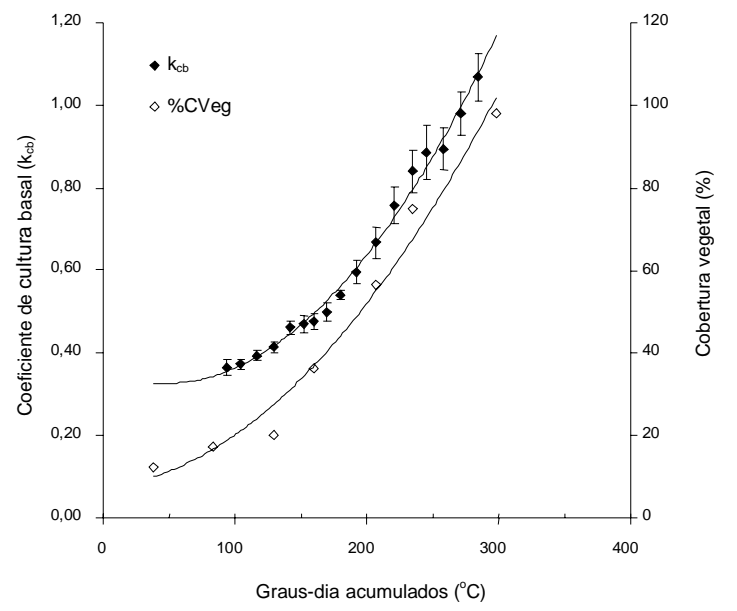

FIG . 6. Coeficiente de cultura basal $\left(k_{\mathrm{cb}}\right)$ e porcentagem de cobertura vegetal $(\% \mathrm{CVeg})$, em razão dos graus-dia acumulados na cultura do feijoeiro, semeada a uma densidade populacional de 50 plantas $\mathrm{m}^{-2}$, em Campinas, 1994. As barras referem-se ao desviopadrão das leituras entre os evapotranspirômetros.

água no solo poucas vezes ter passado de $60 \mathrm{kPa}$, o valor de $\mathrm{k}_{\mathrm{c}}$ foi menor que $\mathrm{k}_{\mathrm{cb}}$, por um curto intervalo.

Nos evapotranspirômetros, o fornecimento contínuo de água para as plantas propiciou uma condição na qual a variação do coeficiente de cultura foi mais diretamente relacionada ao crescimento do feijoeiro. Estes fatos se refletem a boa correlação obtida entre $\mathrm{k}_{\mathrm{cb}}$ e graus-dia acumulados no evapotranspirômetro $\left(\mathrm{r}^{2}=0,97\right)$ sem molhamento da superfície do solo e sem deficiência hídrica, em contraste com a obtida entre $\mathrm{k}_{\mathrm{c}}$ e graus-dia acumulados nos tratamentos no campo de 14 plantas $\mathrm{m}^{-2}$ $\left(\mathrm{r}^{2}=0,81\right)$ e 28 plantas $\mathrm{m}^{-2}\left(\mathrm{r}^{2}=0,78\right)$.

\section{CONCLUSÕES}

1. As relações obtidas entre parâmetros de crescimento da planta e coeficiente de cultura com graus-dia acumulados são altamente significativas, porém fortemente afetadas por fatores mesológicos, como densidade de plantio, suprimento de água e temperatura.

2. As fases de desenvolvimento da cultura não são afetadas pelos tratamentos, demonstrando a efetividade do uso de graus-dia para a predição dos estádios fenológicos da cultura, em diferentes ambientes.

\section{AGRADECIMENTOS}

Ao Fundo de Apoio à Pesquisa (FAEP) da Unicamp, pelo suporte financeiro ao trabalho; ao Instituto Agronômico, através do Centro de Ecofisiologia e Biofísica do IAC, pelo apoio dos técnicos e pesquisadores.

\section{REFERÊNCIAS}

ADAMS, J.E.; ARKIN, G.F.; RITCHIE, J.T. Influence of row spacing and straw mulch on first stage drying. Soil Science of American Journal, Madison, v.40, p.436-442, 1976.

AMOS, B.; STONE, L.R.; BARK, L.D. Fraction of thermal units as the base for an evapotranspiration crop coefficient curve for corn. Agronomy Journal, Madison, v.81, p.713-716, Sept./Oct.1989.

ARRUDA, F.B. Determinação da cobertura do solo durante o ciclo das culturas. Revista Brasileira de Ciência do Solo, Campinas, v.8, p.145-150, 1984.

ARRUDA, F.B. Growth of maize and beans as related to plant density, radiation interception and water stress: a simple model. Davis : University of California, 1987. 195p. Ph.D. Thesis.

ARRUDA, F.B.; TUBELIS, A.; NASCIMENTO, F.J.L. Efeito da temperatura média diária do ar na produtividade do feijoeiro. Pesquisa Agropecuária Brasileira, Brasília, v.15, n.4, p.413-417, abr. 1980.

CAMARGO, O.A.; MONIZ, A.C.; JORGE, J.A.; VALADARES, J.M.A.S. Métodos de análise química, mineralógica e física de solos do Instituto Agronômico de Campinas. Campinas : Instituto Agronômico, 1986. 94p. (IAC. Boletim, 106)

ENCARNAÇÃO, C.R.F. Estudo da demanda hídrica de água do feijoeiro (Phaseolus vulgaris L.) var. Goiano Precoce. Piracicaba: ESALQ, 1980. 62p. Dissertação de Mestrado. 
GUTIERREZ, A.P.; MARIOT, E.J.; CURE, J.R.; RIDDLE, C.S.W.; ELLIS, C.K.; VILLACORTA, A.M. A model of bean (Phaseolus vulgaris L.) growth types I-III: factors affecting yield. Agricultural Systems, Oxford, v.44, p.35-63, 1994.

HOOGENBOOM, G.; WHITE, J.W.; JONES, J.W.; BOOTE, K.J. BEANGRO: a process-oriented dry bean model with a versatile user interface. Agronomy Journal, Madison, v.86, p.182-190, 1994.

KISH, A.J.; OGLE, W.L. Improving the heat unit system in predicting maturity date of snap beans. HortScience, Alexandria, v.15, n.2, p.140-141, 1980.

LAFLEN, J.B.; LANE, L.J.; FOSTER, G.R. WEPP: a new generation of erosion prediction technology. Journal of Soil and Water Conservation, Ankeny, p.3438, Jan./Feb. 1991.

MEDEIROS, G.A. Influência do desenvolvimento do dossel vegetativo sobre o consumo de água e coeficiente de cultura do feijoeiro (Phaseolus vulgaris L.). Campinas : UNICAMP, 1996. 135p. Dissertação de Mestrado.

MEDEIROS, G.A.; ARRUDA, F.B. Adaptação e avaliação de evapotranspirômetros para a obtenção do coeficiente de cultura basal $\left(\mathrm{k}_{\mathrm{cb}}\right)$ do feijoeiro (Phaseolus vulgaris L.). Irriga, Botucatu, v.3, p.137150, 1998

NIELSEN, D.C.; HINKLE, S.E. Field evaluation of basal crop coefficients for corn based on growing degree days, growth stage, or time. Transactions of the ASAE, St Joseph, v.39, n.1, p.97-103, 1996.

OMETTO, J.C. Bioclimatologia vegetal. São Paulo : Agronômica Ceres, 1981. 440p.

PAVANI, L.C. Evapotranspiração e produtividade em feijoeiro comum (Phaseolus vulgaris $\mathrm{L}$. var. Goiano Precoce) sob três níveis de potencial de água no solo. Piracicaba : ESALQ, 1985. 171p. Dissertação de Mestrado.

PIRES, R.C.M.; ARRUDA, F.B.; FUJIWARA, M.; SAKAI, E.; BORTOLETTO, N. Profundidade do sistema radicular das culturas de feijão e trigo sob pivô central. Bragantia, Campinas, v.50, n.1, p.153162,1991

REICHARDT, K. Processos de transferência no sistema solo-planta-atmosfera. Campinas : Fundação Cargill, 1985. 445p.
SAMMIS, T.W.; MAPEL, C.L.; LUGG, D.G.; LANSFORD, R.R.; McGUCKIN, J.T. Evapotranspiration crop coefficients predicted using growing-degree-days. Transactions of the ASAE, St Joseph, v.28, n.3, p.773-780, 1985.

SEPASKHAH, A.R.; ILAMPOUR, S. Effects of soil moisture stress on evapotranspiration partitioning. Agricultural Water Management, Amsterdam, v.28, p.311-323, 1995.

STEGMAN, E.C. Corn crop curve comparisons for the central and northern plains of the U. S. Applied Engineering in Agriculture, St Joseph, v.4, n.3, p.226-233, 1988.

VILLACORTA, A.; GUTIERREZ, A.P.; GUIMARÃES, M.F.; CARVAJAL, B.L.P.; MIGLIORANZA, E. A model of dry bean growth and development: cultivar Carioca. Pesquisa Agropecuária Brasileira, Brasília, v.25, n.3, p.365-370, mar. 1990.

VILLA NOVA, N.A.; OMETTO, J.C. Adaptação e simplificação do método de Penman às condições climáticas do Estado de São Paulo. In: SIMPÓSIO BRASILEIRO DE HIDROLOGIA E RECURSOS HÍDRICOS, 4., Fortaleza, 1981. Anais. Fortaleza : Associação Brasileira de Recursos Hídricos, 1981. v.3., p.281-299.

WALLACE, D.H.; GNIFFKE, P.A.; MASAYA, P.N.; ZOBEL, R.W. Photoperiod, temperature, and genotype interaction effects on days and nodes required for flowering of bean. American Society for Horticultural Science Journal, v.116, p.534$543,1991$.

WANG, J.Y. A critique of the heat unit approach to plant response studies. Ecology, Washington, v.41, n.4, p.785-790, 1960.

WILLIAMS, J.R.; JONES, C.A.; KINIRY, J.R.; SPANEL, D.A. The EPIC crop growth model. Transactions of the ASAE, St Joseph, v.32, n.2, p.497-511, 1989.

WRIGHT, J.L. Crop coefficients for estimates of daily crop evapotranspiration. In: IRRIGATION SCHEDULING FOR WATER \& ENERGY CONSERVATION IN THE 80'S, 1981, Chicago. Proceedings. St Joseph : ASAE, 1981. p.18-26. 